avances

en cienciase ingenierías

II Simposio
Latinoamericano de Aplicaciones Nucleares en la Agricultura

\title{
Evaluación in vivo del efecto genotóxico y citotóxico de los extractos acuosos de dos variedades mutantes de Hibiscus sabdariffa L.
}

\author{
Kristha Paredes Branda', Héctor Nakayama', María Caridad González² y Edith Segovia*1 \\ 'Universidad Nacional de Asunción, Dirección General de Investigación Científica y Tecnológica, \\ Centro Multidisciplinario de Investigaciones Tecnológicas (UNA, DGICT, CEMIT), Laboratorio de \\ Biotecnología, San Lorenzo, Paraguay. \\ ${ }^{2}$ Instituto Nacional de Ciencias Agrícolas (INCA), Mayabeque, Cuba. \\ *Autor para Correspondencia/Corresponding Author, e-mail: edaluz@gmail.com
}

\section{In vivo evaluation of the genotoxic and cytotoxic effect of the aqueous extracts of two mutant varieties of Hibiscus sabdariffa L.}

\section{Resumen}

El Hibiscus sabdariffa (flor de Jamaica, grosella o rosella), conocida además como rosa piola en Paraguay, tiene uso medicinal en gran parte del mundo; en Paraguay se utiliza como antiescorbútico, mientras que en otros países se utiliza como antihipertensivo, antiespasmódico o en el tratamiento de la obesidad, entre otros usos. La radiación gamma es utilizada para la obtención de variedades mejoradas de diferentes especies vegetales; las variedades de grosella Ana Delia y Dogo fueron obtenidas por irradiación Gamma como parte del programa de mejoramiento genético del Instituto Nacional Ciencias Agrícolas (Cuba). El objetivo de este trabajo fue evaluar el efecto genotóxico y citotóxico del extracto acuoso de cálices de ambas variedades de grosella sobre células de médula ósea de ratones tratados con tres concentraciones de cada extracto, vía oral, durante 15 días. El efecto genotóxico fue evaluado mediante la aplicación del ensayo de Micronúcleos. El efecto citotóxico se verifica por el índice obtenido entre la frecuencia de Eritrocitos Policromáticos (eritrocitos jóvenes) y la frecuencia de Eritrocitos Normocromáticos (eritrocitos adultos). Los resultados mostraron que los extractos evaluados, en las concentraciones utilizadas, no tienen un efecto genotóxico o citotóxico en células de médula ósea de ratones tratados por

Editado por /

Edited by:

Gabriela Albán

Recibido /

Received:

$12 / 08 / 2020$

\section{Aceptado /}

Accepted:

24/10/2020

Publicado en línea / Published online: 01/04/2021
15 días con los extractos descritos.

Palabras clave: Citotoxicidad, Eritrocitos Normocromáticos, Eritrocitos Policromáticos, Irradiación gamma, Micronúcleos.

\section{Abstract}

The Hibiscus sabdariffa (Jamaica flower, grosella or rosella), also known as rosa piola in Paraguay, has medicinal use in much of the world; in Paraguay it is used as an antiscorbutic, while in other countries it is used as an antihypertensive, antispasmodic 
or in the treatment of obesity, among other uses. Gamma irradiation is used to obtain improved varieties of different plant species; the Ana Delia and Dogo varieties were obtained by Gamma irradiation as part of INCA genetic improvement program. The objective of this work was to evaluate the genotoxic and cytotoxic effect of the aqueous extract of calyces of both varieties of currant on bone marrow cells of mice treated with three concentrations of each extract, orally, for 15 days. The genotoxic effect was evaluated by applying the Micronucleus test. The cytotoxic effect is verified by the index obtained between the frequency of Polychromatic Erythrocytes (young erythrocytes) and the frequency of Normochromatic Erythrocytes (adult erythrocytes). The results showed that the extracts evaluated, in the concentrations used, do not have a genotoxic or cytotoxic effect on bone marrow cells of mice treated for 15 days with the extracts.

Keywords: Erythrocytes, Gamma radiation, Micronucleus, Normochromatic, Polychromatic erythrocytes.

\section{INTRODUCCIÓN}

El Hibiscus sabdariffa (flor de Jamaica, grosella o rosella), es originaria de Asia [1], aunque otros autores sostienen que es originaria de África y es consumido en varios países de la región, así como el Latinoamérica [2]. En Paraguay es conocido como rosa piola, la infusión del cáliz de la flor se utiliza como antiescorbútico [3] y también se la utiliza para preparar mermeladas [4].

En una descripción del perfil fitoquímico de los cálices de H. sabdariffa se describen la presencia de antocianinas, ácidos orgánicos, polisacáridos y flavonoides, entre otros [2]. En un estudio fitoquímico realizado en dos variedades de $H$. sabdariffa cultivadas en Cuba, se evaluaron la presencia del contenido de los cálices y se registró la presencia de saponinas, compuestos fenólicos, flavonoides, azúcares reductores, aminoácidos, antraquinonas, terpenoides y alcaloides [5].

Se describen varias propiedades medicinales de extracto de esta especie. Se evaluó el extracto acuoso del cáliz en células de tumor de mama y se observó que presenta un efecto citotóxico sobre las mismas [6], en otro ensayo in vitro con células de carcinoma de hígado, pulmón y cáncer colorectal no se observó ningún efecto citotóxico [7]. Se utiliza para el tratamiento de la obesidad [8], tratamiento de la hipertensión e hiperlipidemia [9] y la infusión del cáliz se utiliza como antiescorbútico en Paraguay [3].

El uso de radiación para la obtención de nuevas variedades de especies vegetales es de gran importancia [10]. Las variedades mutantes de H. sabdariffa Ana Delia y Dogo fueron obtenidas por radiación gamma de ${ }^{60} \mathrm{Co}$ (150 Gy y 100 Gy, respectivamente) a partir de semillas de la variedad Yersy. La nueva variedad Ana Delia presentó diferencias en las hojas, tallos, flores y frutos con mejores características y en la selección durante tres años se mantuvieron las características [11]; la variedad Dogo presentó diferencias en el número de ramas, forma de las hojas, cantidad de frutos por planta y peso de los mismos con relación a la variedad de origen [12]. 
Las plantas de uso medicinal deben ser evaluadas en sus posibles efectos secundarios para establecer su inocuidad o su potencial de genotoxicidad [13,14]. Los efectos secundarios de agentes químicos o físicos se pueden evaluar por la técnica de análisis de micronúcleos [15], donde se evalúa la pérdida del material genético por ruptura en el cromosoma (efecto clastogénico) o pérdida de un cromosoma entero (efecto aneugénico), los cuales quedan rezagados en el citoplasma y pueden ser visualizados en una evaluación por microscopía de inmersión; es rápida y relativamente de bajo costo $[16,17]$. En el ensayo de variaciones en las frecuencias de células micronucleadas (MNs) para evaluar el potencial genotóxico, donde se comparan las frecuencias de micronúcleos en las muestras tratadas con las testigo y se evalúan su significancia. También en las muestras obtenidas se evalúa el efecto citotóxico mediante las comparaciones de los ratio entre los eritrocitos jóvenes y adultos observadas o por el porcentaje de eritrocitos jóvenes en el total de eritrocitos [18,19].

El objetivo de este trabajo fue evaluar los efectos genotóxicos y citotóxicos de los extractos acuosos de las nuevas variedades de $H$. sabdariffa descritas, en células de médula ósea de ratones.

\section{MATERIALES Y MÉTODOS}

\section{Plantas y extractos}

Extracto acuoso de hojas de H. sabdariffa, variedades Ana Delia y Dogo y los cálices de los frutos fueron obtenidos en el INCA. Se sembraron las variedades en suelo ferralítico rojo compactado, y al ponerse las hojas de las planas amarillas se cosecharon lo cálices de los frutos y estos se pusieron a secar en una sala a $24^{\circ} \mathrm{C}$ por 10 días (comunicación oral de la Dra. Caridad González). Se utilizaron 20 cálices deshidratados de cada variedad (Ana Delia $16.4 \mathrm{~g}$ y Dogo 11.5g). Por separado, los cálices se trituraron y se llevaron a ebullición en $2000 \mathrm{ml}$ de agua potable. Se procedió a filtrar cada infusión con algodón y papel de filtro Whatman, se liofilizó y se conservó a $-20^{\circ} \mathrm{C}$ hasta el momento de su uso. Se utilizaron 20 flores de cada variedad.

\section{Animales y tratamientos}

Los ratones fueron adquiridos al Instituto de Investigaciones en Ciencias de la Salud (IICS) de la UNA y fueron aclimatados por 7 a 10 días. Se utilizaron ratones Swiss albinos, machos, de entre 8 a 12 semanas de vida, que fueron mantenidos en la Sala de Mantenimiento de Animales del laboratorio de Biotecnología del CEMIT- UNA, con temperatura controlada y con agua y comida ad libitum, con 12 horas de luz y 12 horas a oscuras. Los animales fueron distribuidos aleatoriamente en grupos de cinco animales por tratamiento. El tratamiento consistió en administrar por vía oral, mediante el uso de una cánula intragástrica, tres concentraciones diferentes 0.53 mg; 0.8 mg y 1.6 mg de Ana Delia por kg de animal; 0.4 mg, $0.6 \mathrm{mg}$ y $0.12 \mathrm{mg}$ por $\mathrm{kg}$ de animal del extracto acuoso de la variedad Dogo, durante 15 días. Para calcular las concentraciones, se consideró el uso de los cálices para una persona de $60 \mathrm{~kg}$ de peso y estimando el peso de los animales de 33 gramos, en promedio. Las concentraciones utilizadas de cada variedad fueron diferentes debido a que las flores de la variedad Dogo eran de tamaño 
menor y se tomó como referencia la cantidad de flores por litro de agua, no el peso de las mismas. Para el control positivo se utilizó el antineoplásico ciclofosfamida (CP) y se utilizaron los mismos datos para evaluar ambas variedades.

\section{Ensayo de micronúcleos (MNs)}

Para este ensayo se utilizó el método de Schmid, 1975[15]. Los animales fueron tratados vía oral, por 15 días, luego fueron sacrificados por dislocación cervical. Se extrajo la médula ósea de los fémures de cada animal, se lavaron con Suero Bovino Fetal y se prepararon las muestras haciendo el frotis en portaobjetos limpios y secos. Se fijaron las muestras con metanol $100 \%$, por cinco minutos y se tiñeron las mismas con colorante Giemsa al 5\%, diluido en agua destilada estabilizada con tampón. Se analizaron las muestras en microscopio óptico de inmersión y se contaron 1000 eritrocitos policromáticos (EPCS) incluyendo los que presentaron micronúcleos (EPCMNs), por animal tratado. Para el análisis de citotoxicidad se analizaron las mismas muestras del ensayo de MNs, donde 500 eritrocitos fueron contados por animal tratado, incluyendo los eritrocitos maduros (eritrocitos normocromáticos-ENC) y jóvenes (eritrocitos policromáticos-EPC) y se analizaron las proporciones de los mismos en cada muestra; las desviaciones en las proporciones son indicativos de toxicidad.

\section{Análisis estadístico}

Los datos fueron procesados mediante el software de análisis estadístico EpilnfoTM versión 3.5.1. Ensayo estadístico ANOVA ( $p<0.05)$.

\section{RESULTADOS}

En la evaluación del extracto acuoso de la variedad Ana Delia se observó que la frecuencia de células micronucleadas en médula ósea de ratones tratados con el extracto no fue significativa, comparada con la frecuencia del control negativo. Tampoco fue significativo el efecto citotóxico, evaluado por la ratio entre eritrocitos jóvenes y maduros. El control positivo ciclofosfamida $\left(50 \mathrm{mg} \mathrm{kg}^{-1}\right)$ utilizado resultó significativo en los análisis de genotoxicidad y citotoxicidad (Tabla 1).

Tabla 1. Frecuencia de eritrocitos policromáticos micronucleados, en médula ósea de ratones machos tratadas con tres concentraciones de H. sabdariffa de la variedad mutante Ana Delia.

\begin{tabular}{|c|c|c|c|}
\hline Grupo experimental & Total EPC & Total EPCMN & Ratio EPC/ENC \\
\hline CTL- & 5.000 & 3 & 1.60 \\
\hline $0.53 \mathrm{mg}$ & 5.000 & 5 & 1.61 \\
\hline $0.8 \mathrm{mg}$ & 5.000 & 4 & 1.57 \\
\hline $0.16 \mathrm{mg}$ & 5.000 & 4 & 1.62 \\
\hline $\mathrm{CP}$ & 5.000 & $38^{*}$ & $1.16^{*}$ \\
\hline
\end{tabular}


Al evaluar la frecuencia de micronúcleos en las células de médula ósea de los ratones con los tratamientos realizados con el extracto acuoso de la variedad Dogo por 15 días, no se encontró un aumento significativo del número total de micronúcleos. Los tratamientos no presentaron un efecto genotóxico. Al evaluar las ratios entre eritrocitos jóvenes y maduros en células de médula ósea de los ratones tratados, no fueron significativos cuando fueron comparados con los resultados obtenidos en los ratones del control negativo. Los resultados obtenidos en los ratones del control positivo fueron estadísticamente significativos tanto en la evaluación de genotoxicidad como de citotoxicidad (Tabla 2).

Tabla 2. Frecuencia de eritrocitos policromáticos micronucleados, en médula ósea de ratones machos tratadas con tres concentraciones de H. sabdariffa de la variedad mutante Dogo.

\begin{tabular}{|c|c|c|c|}
\hline Grupo experimental & Total EPC & Total EPCMN & Ratio EPC/ENC \\
\hline CTL- & 5.000 & 3 & 1,60 \\
\hline $0,4 \mathrm{mg}$ & 5.000 & 4 & 1,55 \\
\hline $0,6 \mathrm{mg}$ & 5.000 & 5 & 1,63 \\
\hline $012 \mathrm{mg}$ & 5.000 & 5 & 1,59 \\
\hline CP & 5.000 & $38^{*}$ & $1,16^{*}$ \\
\hline
\end{tabular}

EPC: eritrocito policromático; EPCMNs: eritrocitos policromático micronucleado; ENC: eritrocito normocromático; CP: ciclofosfamida. Datos procesados por el software de análisis estadístico EpiinfoTM versión 3.5.1.*Ensayo estadístico ANOVA $(p<0.05)$

\section{DISCUSIÓN}

Las especies vegetales utilizadas como alimentos o de uso medicinal pueden ser mejoradas con el uso de la energía atómica, como en el mejoramiento de patata, donde irradiaron enredaderas de Ipomoea batatas Lam. (Patata dulce) con rayos gamma, con 2 y 10 Gy, por 20 horas; y, aunque no obtuvieron el número esperado de ejemplares con la característica fenotípica deseada, el estudio pudo ser la base para otros ensayos [20]. Irradiando semillas de Cicer arietinum L con ${ }^{60} \mathrm{Co}$ (280 Gy) se obtuvieron poblaciones mutantes con características fenotípicas, como días de floración, altura de la planta y otros, con diferencias significativas [21], también se utilizó en el mejoramiento de cítricos [22].

La especie vegetal $H$. sabdariffa tiene amplio uso como planta medicinal [1]. Se ha descrito su efecto protector en células de médula ósea de ratones tratados con el extracto acuoso de la planta y sodio arsenito, reduciendo la frecuencia de micronúcleos en los animales tratados con el extracto [23]. Las evaluaciones de genotoxicidad se realizan en varios agentes, para determinar su inocuidad, como fármacos [24], extractos de especies agronómicas como el Allium sp. [25], principios activos de plantas [16] o extractos de plantas de uso medicinal [14]. No se ha encontrado en la literatura especializada evaluaciones de los efectos secundarios de variedades mutantes de plantas medicinales. En este trabajo se evaluó el extracto acuoso de dos variedades mutantes de $\mathrm{H}$. sabdariffa mediante el ensayo de micronúcleos [15], ensayo más utilizado en la evaluación de la genotoxicidad de extractos de plantas [14]. Considerando el uso como planta medicinal de la H. sabdariffa no modificada y con el fin de evaluar si estas nuevas variedades no 
presentan un potencial genotóxico, se evaluaron tres concentraciones de cada variedad citada. Al comparar las frecuencias de micronúcleos en células de médula ósea de ratones de los grupos tratados con los extractos de Ana Delia y Dogo, estas frecuencias no fueron significativas. En la evaluación de citotoxicidad los resultados no fueron significativos, los tratamientos no indujeron citotoxicidad en las células de médula ósea.

\section{CONCLUSIÓN}

Los extractos acuosos de las variedades mutantes Ana Delia y Dogo de H. sabdariffa no presentaron efectos genotóxicos o citotóxicos en células de médula ósea de ratones, en las condiciones utilizadas. Se sugieren otros ensayos con estas variedades para determinar su inocuidad o el potencial tóxico o genotóxico, como parte de las medidas preventivas en el uso de una planta consumida en varias regiones del mundo.

\section{AGRADECIMIENTOS}

Al Ing Agr. Carlos Emilio Mussi, por su invalorable apoyo constante y disposición a colaborar desinteresadamente. Este trabajo experimental fue aprobado por el Departamento de Investigación del Centro Multidisciplinario de Investigaciones Tecnológicas (CEMIT, DGICT, UNA).

\section{CONTRIBUCIONES DE LOS AUTORES}

Kristha Noemí Paredes Branda: adquisición de datos de laboratorio (tratamiento de los animales, obtención de las muestras y análisis citogenético) y análisis estadístico de los mismos. Validación de los resultados y revisión del manuscrito. María Caridad González Cepero obtuvo las muestras de cáliz de H. sabdariffa y concibieron con Héctor Nakayama la investigación. Edith Segovia coordinó el trabajo de laboratorio, proveyó los reactivos y redactó el manuscrito. 


\section{REFERENCIAS}

[1] Riaza, G., \& Choprab, R. (2018) A review on phytochemistry and therapeutic uses of Hibiscus sabdariffa L. Biomedicine \& Pharmacotherapy 102 (2018) 575-586. Doi: doi.org/10.1016/j.biopha.2018.03.023

[2] Da-Costa-Rocha, I., Bonnlaender, B., Sieversc, H., Ivo Pischel, I., \& Heinrich, M. (2014) Hibiscus sabdariffa L. - A phytochemical and pharmacological review. Food Chemistry 165 (2014) 424-443.

[3] Pin, A., González, G., Marín, G., Céspedes, G., Cretton, S., Christen, P., \& D. Roguet, (2009). Plantas Medicinales del Jardín Botánico de Asunción. 1a edición. Asunción. p. 157-339.

[4] ABC 2011. https://www.abc.com.py/articulos/rosella-268323.html. Consultado en junio 2020.

[5] Brown, F., González, J., \& Monan, M. (2019) Pharmacognostic, Physicochemical and Phytochemical Analysis of Two Cultivars from Hibiscus sabdariffa L. in Cuba. Open Access Library Journal, 6, 1-8. Doi: 10.4236/oalib.1105894.

[6] Nguyen, C., Baskaran, K., Pupulin, A., Ruvinov, I., Zaitoon, 0., Grewal, S., Scaria, B., Mehaidli, A., Vegh, C., \& Pandey, S. (2019). Hibiscus flower extract selectively induces apoptosis in breast cancer cells and positively interacts with common chemotherapeutics. BMC complementary and alternative medicine, 19(1), 98. Doi: https://doi.org/10.1186/ s12906-019-2505-9.

[7] Maciel, L. G., do Carmo, M. A. V., Azevedo, L., Daguer, H., Molognoni, L., \& de Almeida, M.M. (2018). Granato. Food Chem Toxicol. 2018 Mar;113:187-197. Doi: 10.1016/j.fct.2018.01.053.

[8] Ojulari, O.V., Lee, S.G., \& Nam, J. 0. (2019). Beneficial Effects of Natural Bioactive Compounds from Hibiscus sabdariffa L. on Obesity. Molecules. Jan 8;24(1):210. Doi: 10.3390/molecules24010210.

[9] Hopkins, A.L., Lamm, M. G., Funk, J. L., \& Ritenbaugh, C. (2013). Hibiscus sabdariffa L. in the treatment of hypertension and hyperlipidemia: a comprehensive review of animal and human studies. Fitoterapia, 85:84-94. Doi: 10.1016/j. fitote.2013.01.003.

[10] Kuranouchi, T., Kumazaki, T., Kumagai, T., \& Nakatani, M. (2016). Breeding erect plant type sweetpotato lines using cross breeding and gamma-ray irradiation. Breed Sci, (3):456-61. doi: 10.1270/jsbbs.15134.

[11] González Cepero, M. C., Trujillo Gil, D., Terán Vidal, Z., \& Valentín, S.A. (2011). Informe de nuevas variedades ANA DELIA, mutante de flor de Jamaica (Hibiscus sabdariffa) obtenido en Cuba mediante el empleo de inducción de mutaciones con rayos Gamma de ${ }^{60} \mathrm{CO}$. Cultivos Tropicales, 32 (4), p. 27.

[12] González Cepero, M. C. (2015). Reporte de nuevo cultivar Dogo, nuevo cultivar cubano de Flor de Jamaica (Hibiscus sabdariffa, L) obtenido por inducción de mutaciones con rayos Gamma ${ }^{60} \mathrm{CO}$. Cultivos Tropicales, 36 (4), pp. 133.

[13] OECD: 2016 https://one.oecd.org/document/C(2016)103/en/pdf.. Consultado en junio 2020.

[14] Sponchiado, G., Adam, M.L., Silva, C. D., Soley, B. S., de Mello-Sampayo, C., Cabrini, D. A., Correr, C. J., \& Otuki, M. F. (2016). Quantitative genotoxicity assays for analysis of medicinal plants: A systematic review. J Ethnopharmacol, 178:289-96. Doi: 10.1016/j.jep.2015.10.026.

[15] Schmid, W. (1975). The micronucleus test for cytogenetic analysis. Chemical Mutagens 4.31.

[16] Chen, R., Zhou, C., Cao, Y., Xi, J., Ohira, T., He, L., Huang, P., You, X., Liu, W., Zhang, X., Ma, S., Xie, T., Chang, Y., \& Luan, Y. (2020) Assessment of Pig-a, Micronucleus, and Comet Assay Endpoints in Tg.RasH2 Mice Carcinogenicity Study of Aristolochic Acid I. Environ Mol Mutagen, 61(2):266-275. doi: 10.1002/em.22325.

[17] Ortega, Y. H., Mosquera, D.M., Galapththi, S., Santos, B.T., González, G.S., Foubert, K., Pieters, L., Calderon, P.B., \& Apers, S. (2016). Safety assessment of a traditionally used extract from leaves of Boldoa purpurascens. Ethnopharmacol, 192:302-308. Doi: 10.1016/j.jep.2016.07.009.

[18] Attia, S.M. (2007). Chromosomal composition of micronuclei in mouse bone marrow treated with rifampicin and nicotine, analyzed by multicolor fluorescence in situ hybridization with pancentromeric DNA probe. Toxicology, 235, 112-118. Doi:10.1016/j.tox.2007.03.011.

[19] Otabea, A., Ohtaa, F., Takumia, A., \& Lynch, B. (2019). Mutagenicity and genotoxicity studies of aspartame. Regulatory Toxicology and Pharmacology, 103, 345-35. Doi: 10.1016/j.yrtph.2018.01.023.

[20] Kuranouchi, T., Kumazaki, T., Kumagai, T., \& Nakatani, M. (2016). Breeding erect plant type sweet potato lines using cross breeding and gamma-ray irradiation. Breed Sci., 66(3):456-61. Doi: 10.1270/jsbbs.15134. 
[21] Amri-Tiliouine, W., Laouar, M., Abdelguerfi, A., Jankowicz-Cieslak, J., Jankuloski, L., \& Till, B.J. (2018). Genetic Variability Induced by Gamma Rays and Preliminary Results of Low-Cost TILLING on $\mathrm{M}_{2}$ Generation of Chickpea (Cicer arietinum L.). Front. Plant Sci, 9:1568. Doi: 10.3389/fpls.2018.01568.

[22] Talón, M., López-García, A., Terol, J., Cercós, M., Ibañez, V., Herrero-Ortega, A., Muñoz-Sanz, J.V., Colmenero-Flore, J.M., Arbona, V., Estornell, L.H., Carbonell, J., Concesa, A., Dopazo, J., \& Tadeo, F.R. (2011). Citruseq: una proximación genómica a la mejora de los cítricos. Levante Agrícola 405: 73-78.

[23] Adetutu, A., Odunola, 0. A., Owoade, A., Adeleke, 0. A., \& Amuda, S. A. (2004). Anticlastogenic Effects of Hibiscus sabdariffa Fruits against Sodium Arsenite-induced Micronuclei Formation in Erythrocytes in Mouse Bone Marrow. Phytotherapy Research, 18, 862-864. Doi: 10.1002/ptr.1554.

[24] Brock, W. J., \& Bell, T. A. (2012). The in vitro and in vivo genotoxicity of benzocaine: a brief communication. J Toxicol., 31(3):222-7. Doi: 10.1177/1091581812442230.

[25] Mellado-García, P., Puerto, M., Prieto, A. I., Pichardo, S., Martín-Cameán, A., Moyano, R., Blanco, A., \& Cameán, A.M. (2016). Genotoxicity of a thiosulfonate compound derived from Allium sp. intended to be used in active food packaging: In vivo comet assay andmicronucleus test. Mutation Research 800, 1-11. 